\title{
Effect of Sequence of Infection of Maize with Viruses Causing Maize Lethal Necrosis on Disease Development
}

\author{
Roy Gitonga Kiambi ${ }^{1,3 *}$ Douglas Watuku Miano ${ }^{2}$ John Maina Wagacha ${ }^{1} \quad$ Ann Wangai $^{3}$ \\ 1. School of Biological Sciences. P. O. Box 30197-00100 Nairobi, University of Nairobi, Kenya \\ 2.Department of Plant Science and Crop Protection, University of Nairobi, P. O. Box 29053-00625, Kangemi, \\ Nairobi, Kenya \\ 3.Kenya Agricultural and Livestock Research Organization, P. O. Box 14733-00100 Nairobi
}

\begin{abstract}
Maize is the main staple food crop in Kenya. However, its production is constrained by maize lethal necrosis (MLN) disease, a result of dual infection of maize plants with Maize chlorotic mottle virus (MCMV) and a potyvirus Sugarcane mosaic virus (SCMV). In the field, infection by the two viruses is independent rather than occurring simultaneously since MCMV and SCMV are transmitted by different vectors. This study aimed at evaluating the effect of sequence of infection of maize by the two viruses causing MLN disease in the greenhouse. Two susceptible maize hybrids (DUMA 43 and H614D) were each infected with either of the two viruses first and later inoculated with the second virus. The plants were assessed for disease development weekly over a period of 2 months. Symptoms were significantly $(\mathrm{p}=0.05)$ more severe in maize plants initially infected with MCMV followed by SCMV, resulting in rapid death of plants compared to plants infected with SCMV followed by MCMV. The results indicate that severity of symptoms is influenced by the sequence of infection of maize plants with the causal agents of MLN disease. More remarkable was the synergism observed in maize plants pre-inoculated with SCMV followed by MCMV that had a slow manifestation of MLN disease symptoms. The results suggest that initial infection by SCMV may result in maize plants developing some levels of resistance resulting in initial suppression of MCMV infection. This study contributes to the understanding of resistance mechanisms exhibited by the plants during MLN disease development.
\end{abstract}

Keywords: Maize, MCMV, SCMV, Synergism, Sequence of infection

DOI: $10.7176 / \mathrm{JNSR} / 9-8-06$

Publication date: April $30^{\text {th }} 2019$

\section{Introduction}

Maize (Zea mays) is the backbone of food security in Kenya. Large as well as small-scale farmers produce the crop and a large percentage of the population depends on maize farming for income generation (Manje et al. 2015). Maize lethal necrosis (MLN) disease, first reported in 2011 in the Rift Valley, is a serious disease of maize in Kenya (Wangai et al. 2012). The disease results in chlorotic mottling of the leaves, severe stunting, often leading to plant death. Maize lethal necrosis is attributed to a synergistic interaction between Maize chlorotic mottle virus (MCMV, genus Machlomovirus) and potyviruses infecting maize, most frequently Sugarcane mosaic virus (SCMV). Identification of maize germplasm with resistance to MLN disease and the viruses involved in the disease complex is generally by observation of symptoms in the field (Manje et al. 2015). Single infections with either MCMV or SCMV especially at early stages of infection are often inconspicuous and resemble physiological disorders. Such infections cause only mild mosaic or mottling symptoms and a moderate reduction in plant growth. Plants infected with SCMV alone usually show systemic infection of the whole plant, mild mosaic observed on the leaves and sometimes the stems. The whole plant is stunted and yield is moderately affected. Symptoms vary widely depending on host genotype and time of infection. Infection of plants with only MCMV results in various symptoms, including moderate fine chlorotic spots, which appear first on the youngest leaves, which coalesce and develop into broad chlorotic stripes along the veins. These chlorotic stripes contrast with dark green tissue when observed against the light (Lommel et al. 1991). In some cases, infection of the maize plant with MCMV may also cause severe symptoms. Plants affected with MLN disease appear stunted and show a general chlorosis, leaf bleaching and necrosis.

When studying MLN disease, plants are usually inoculated with MCMV and SCMV at the same time. However, since SCMV is transmitted by aphids (including Aphis gossypii and Myzus persicae) while MCMV is transmitted mostly by corn thrips (Frankliniella williamsi) (Cabanas et al. 2013), simultaneous infection is also unlikely to occur in nature. A viral disease caused by the interaction of two independent viruses is characterized by dramatic increase in symptoms and accumulation of one of the co-infecting viruses (Zhou et al. 2017). The presence of any virus changes the environment in which it thrives, and viruses interact when they encounter one another in a common host plant. Synergistic interactions between viruses occur in diverse ways and have varying effects to the plants. One virus may cause, directly or indirectly, an increase and/or decrease in replication of the other virus (Begon and Mortimer, 1986) resulting in plant developing some form of resistance while another may break down resistance. This study was therefore carried out to investigate whether sequence of infection of the 
maize plants by the two viruses has any effect on symptom development and/or result in enhanced or reduced disease severity.

\section{Materials and Methods}

\subsection{Virus Isolates used in the Study}

The Sugarcane mosaic virus (SCMV) and Maize chlorotic mottle virus (MCMV) isolates used in the study were originally obtained from infected maize plants collected in Bomet County, in the South Rift-Valley Region of Kenya and propagated in susceptible maize variety H614D, which also served as the inoculum source. The isolates were maintained in separate secluded greenhouses to avoid contamination.

\subsection{Experimental Design and Layout}

The experiments were carried out in two cropping seasons, during the months of May to July and August to November 2016. Two maize varieties, H614D and Duma 43, which are extensively grown in most parts of Kenya, were used for the study. Pots, $45 \mathrm{~cm}$ in diameter, were filled with sterilized soil to approximately three quarters full and mixed with Diammonium Phosphate (DAP 18:46:0) fertilizer at a rate of five grams per plant. The pots were watered to moisten the soil for ease in planting. Five seeds were planted in each pot at a depth of about $2.5 \mathrm{~cm}$ below the soil surface. The experimental design used was a completely randomized design (CRD) consisting of four replications per treatments and three plants in each replication. The pots were watered every morning and after emergence watering was done carefully every other day. The plants were thinned to three per pot at two leaves stage to ensure no overcrowding of plants during development. The plants were fertilized with calcium ammonium nitrate (CAN 26\%) fertilizer at approximately 2.5 grams per plant at vegetative stage eight (V8). Virus inoculum for either of the viruses was prepared by grinding infected maize leaves with mortar and pestle in buffer in the ratio of 1:10 (w/v) of $0.1 \mathrm{M}$ phosphate buffer $\mathrm{pH} 7.0\left(\mathrm{KH}_{2} \mathrm{PO}_{4}-4.8 \mathrm{~g}, \mathrm{~K}_{2} \mathrm{HPO}_{4}-10.8 \mathrm{~g}, \mathrm{Na}_{2} \mathrm{SO}_{3}-1.26 \mathrm{~g}\right.$ in 1 liter of $\left.\mathrm{dH}_{2} \mathrm{O}, \mathrm{pH} 7.4\right)$. Plant debris were removed by filtering the extracts through a muslin cloth. Each group of maize plants was inoculated with either SCMV or MCMV at V4 growth stage. After first inoculation with the initial virus, plants were allowed to grow for seven days before the second virus was introduced to have a combination sequence of SCMV followed with MCMV (SCMV + MCMV), MCMV followed with SCMV (MCMV + SCMV) to achieve MLN. Plants inoculated with the two viruses at the same time (MLN) and plants inoculated with a single virus were also established as controls.

\subsection{Disease assessment and data analysis}

Symptom severity was assessed and recorded based on a scale of 1-5 adopted from Gowda et al. (2015) where 1 $=$ no symptom, $2=<10 \%$ of plant leaf surface showing symptoms, $3=10-30 \%$ plant leaf surface showing symptoms, $4=30-50 \%$ of plant leaf surface showing symptoms, $5=>50 \%$ of plant leaf surface showing symptoms. Analysis of variance (ANOVA) was used to determine the statistical significance of differences among treatments for the two viruses using GeneStat ${ }^{\circledR} 2015$ (v15.1) at 5\% level of probability. Area Under Disease Progress Curve (AUDPC) scores were calculated for each virus and virus combination on individual plants using the midpoint rule method according to Campbell \& Madden (1990) based on the formula:

AUDPC $=\sum_{\mathrm{i}=1}^{\mathrm{n}-1}\left[\left(\mathrm{t}_{\mathrm{i}+1}-\mathrm{t}_{\mathrm{i}}\right)\left(\mathrm{y}_{\mathrm{i}}+\mathrm{y}_{\mathrm{i}+1}\right) / 2\right]$

Where " $t$ " is time in days of each reading, " $y$ " is the percentage of affected foliage at each reading and " $n$ " is the number of readings.

\subsection{Results}

\subsection{Disease incidence and severity}

Similar trends in disease development were observed in both experiments (May - July and August - November 2016) were similar in the two maize varieties used in the study. Treatments consisting of SCMV alone, SCMV+MCMV, and MLN had symptom expression with a severity score of 2 within the first seven days post inoculation (dpi) in the two maize varieties (Fig. 1 and Fig. 2). Treatments with MCMV alone or MCMV + SCMV had no symptom manifestation in the first $7 \mathrm{dpi}$. However, symptoms rapidly developed from mosaic to tissue necrosis with severe plant stunting and death 21 dpi for MLN and MCMV +SCMV with a severity score of 4.5 (Fig. 1 and Fig. 2) while the single infections of MCMV and SCMV showed mild symptoms (Fig. 3). The first systemically infected leaves of co-infection became highly chlorotic at 9 dpi and developed necrotic areas at 10 dpi while the leaves of SCMV or MCMV single infection showed consistent mosaic or chlorotic symptom by 10 dpi. The systemic symptoms caused by multiple viruses were initially similar to those of single virus infections. At 14 dpi, a symptom rating score of 4 was recorded for MLN. At 18 dpi, symptoms observed in the MCMV + SCMV inoculated plants were significantly more severe than all other treatments except MLN. As of 30 dpi, the symptoms observed on maize plants initially infected with SCMV + MCMV were also significantly more severe than those for singly infected plants (Fig. 4). At 40 dpi, maize plants initially inoculated with MCMV followed by SCMV had more severe symptoms leading to a 'dead heart' (Fig. 5) compared to maize plants inoculated initially 
with SCMV followed by MCMV but less than for those inoculated with MLN (Fig. 1 and Fig. 2).

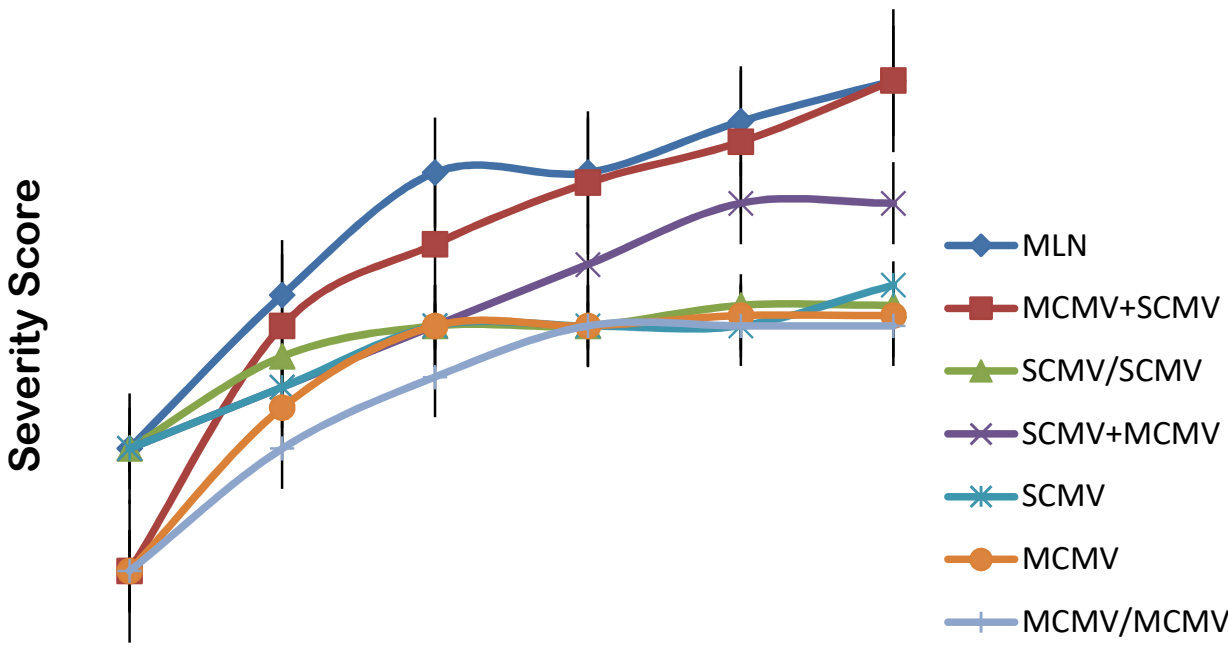

Assesment period (Days)

Figure 1. Development of MLN disease symptoms in plants initially infected with Sugarcane mosaic virus (SCMV), followed by Maize chlorotic mottle virus (MCMV) and plants initially infected with MCMV, followed by infection with SCMV in maize variety DUMA 43. Bars indicate standard error of the means.

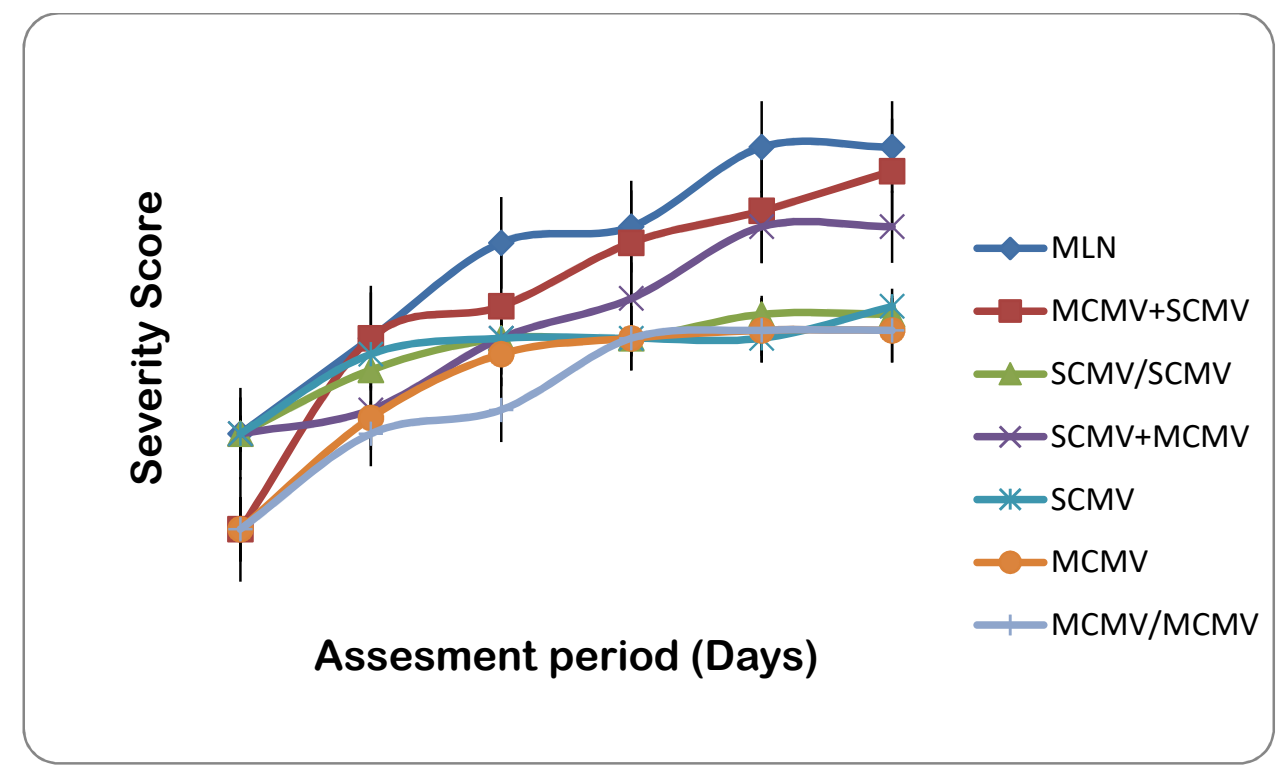

Figure 2. Development of MLN disease symptoms in plants initially infected with Sugarcane mosaic virus (SCMV) followed by Maize chlorotic mottle virus (MCMV) and plants initially infected with MCMV, followed by infection with SCMV and controls on maize variety H614D. Bars indicate standard error of the means. 


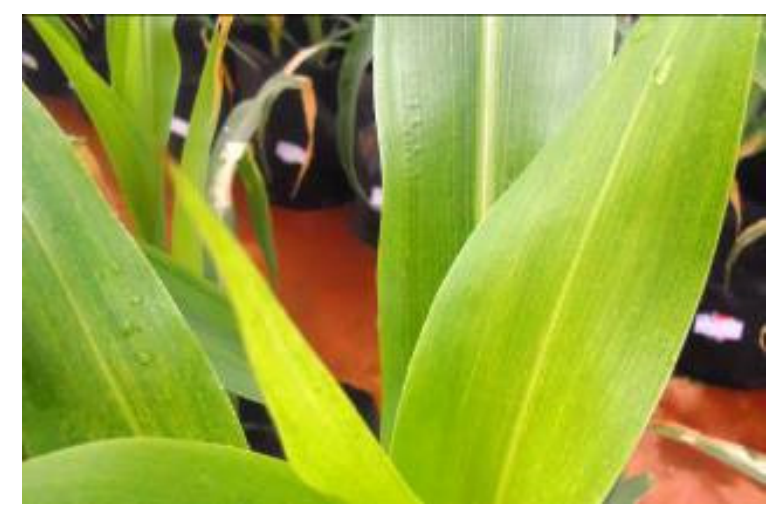

Figure 3. Mild mosaic and mottling symptoms on maize with single infection with Maize chlorotic mottle virus (MCMV).

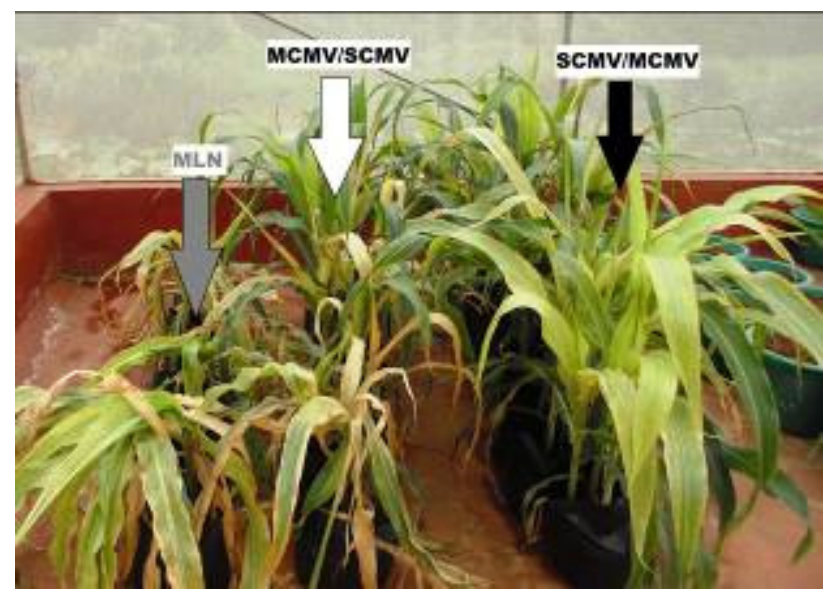

Figure 4. Maize plants showing maize lethal necrosis (MLN) disease symptoms based on sequence of infection.
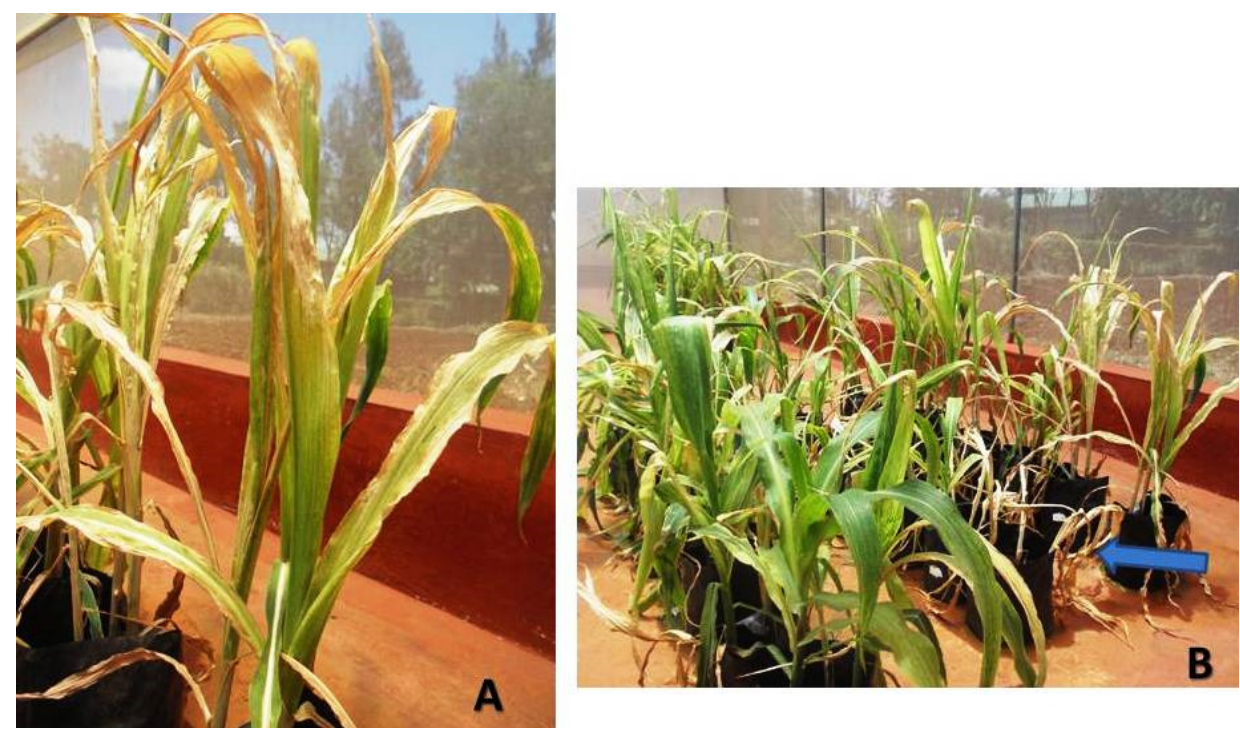

Figure 5. $\mathrm{A}=$ Dead heart symptom $\mathrm{B}=$ Maize plants showing maize lethal necrosis (MLN) disease symptoms with excessive chlorotic mottling with leaf necrosis, arrow showing complete plant necrosis.

\subsection{Area under disease progress curve}

The two maize genotypes used in this study had similar reaction to infection by either of the viruses or their combinations as indicated by the Area Under Disease Progress Curve (AUDPC) (Fig. 6). Maize plants inoculated with MLN at the V4 growth stage had the highest mean AUDPC value for the observation period was recorded at 137.7 and 139.4 for H614D and DUMA 43, respectively. The lowest mean AUDPC scores recorded was 86.61 in H614D and 88.1 in DUMA 43 for MCMV treatment. AUDPC scores for MCMV+SCMV and MLN were 
significantly higher to those of SCMV+MCMV, indicating a delay in symptom development in the co-infection.

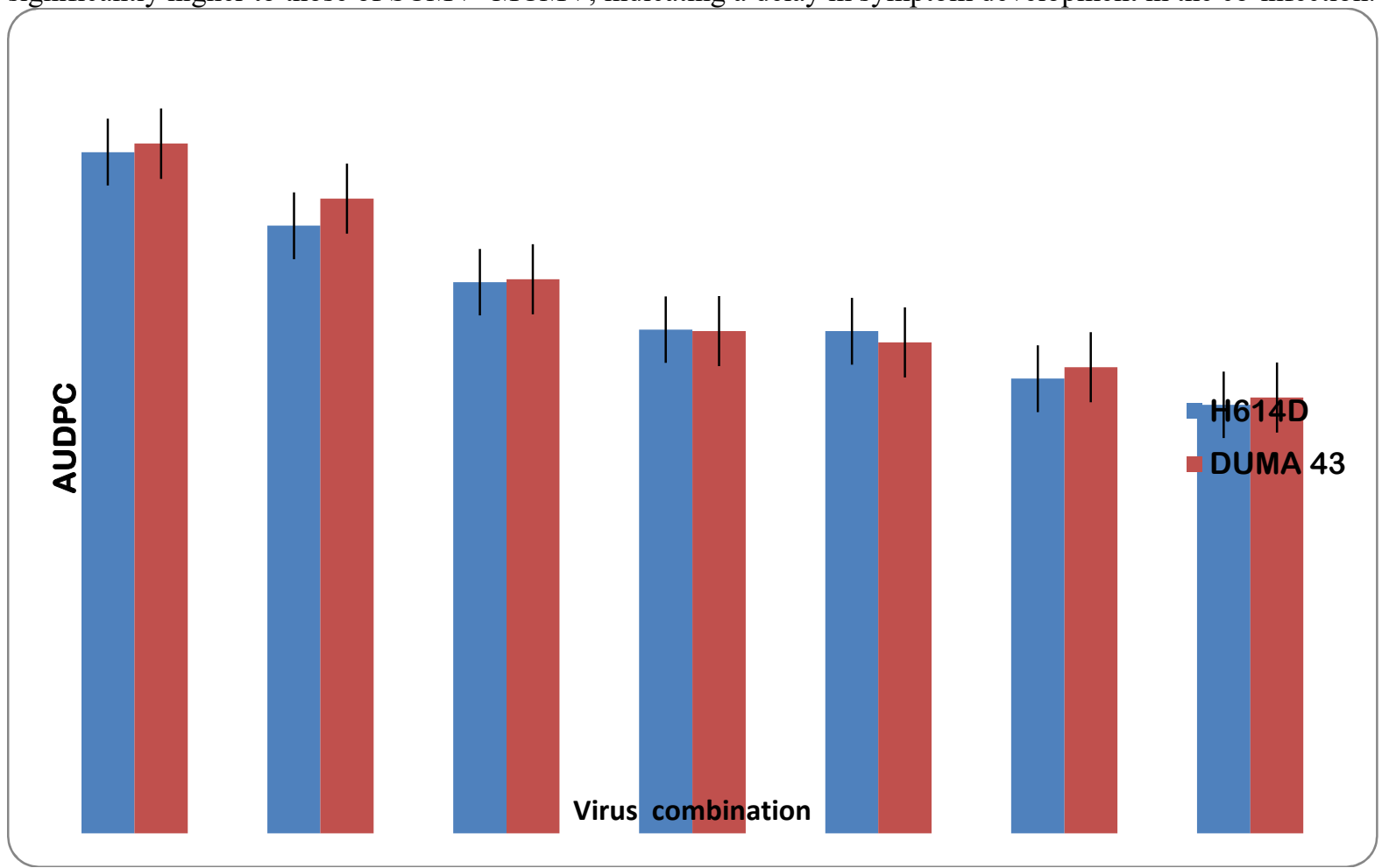

Figure 6. Mean area under disease progress curve (AUDPC) scores for maize plants in inoculated with different virus combinations used from disease severity ratings for two maize varieties. Bars indicate standard error of the means.

\section{Discussion}

In this study, maize seedlings at the fourth leaf stage were initially inoculated with SCMV or MCMV then followed with MCMV or SCMV after seven days to have a sequence combination of SCMV + MCMV and MCMV + SCMV. All plants inoculated with the combination of the two viruses eventually developed into MLN disease, indicating a synergistic interaction regardless of the sequence of infection. Symptoms were first observed in plants initially inoculated with SCMV alone and later in combination with MCMV compared to those initially inoculated with MCMV alone and later in combination with SCMV. However, the plants inoculated with MCMV first and later with SCMV developed symptoms rapidly, which later led to a rapid death of plants unlike the slow and mild manifestation of symptoms expression in the maize plants infected with the individual viruses (SCMV and MCMV).

Similar patterns in other synergistic diseases have been observed with an increase in symptom severity and an increase in viral concentration involving potyviruses (Rochow \& Ross 1955; Goodman \& Ross 1974a, b; Calvert \& Ghabrial 1983; Goldberg \& Brakke 1987; Vance 1991; Anjos et al. 1992; Vance et al. 1995; Anderson et al. 1996). In plants, synergistic interactions among independent viruses in mixed infections have been well studied (Matthews 1991; Syller 2012) but the mechanism of interaction between MCMV and SCMV that result in MLN disease remains unclear. Maize plants with resistance to virus infection are primarily controlled by posttranscriptional gene silencing (PTGS) (Incarbone \& Donoyer, 2013). RNA silencing as a mechanism of plant virus resistance and the suppression of such silencing by viruses are well known (Waterhouse et al. 2002; Voinnet 2005). The surveillance mechanism in the defence against viruses in plants can trigger the production of virus derived small interfering RNAs (vsiRNAs) in virus-infected plant cells. It is suggestive that plants first inoculated with SCMV then followed by MCMV induce RNA silencing mechanism since symptoms induced by SCMV alone are generally mild, thus relatively slow symptoms development in the SCMV +MCMV infected plants. This may indicate that there is an inclination to SCMV RNAs for RNA silencing, which accumulates more S-vsiRNAs than M-vsiRNAs (Xia et al., 2016). In maize plants co-infected with MCMV and a potyvirus (such as SCMV), MCMV concentrations are 3 to 11 times higher than in plants infected with MCMV alone (Goldberg and Brakke 1987; Scheets 1998). It is indicative that, if the plants are prior infected with MCMV, the SCMV resistance mechanism appears to be already suppressed, so that when plants are later infected with SCMV, severe symptoms develop quickly. The striking increase in symptom expression in MCMV/SCMV plants, in part may be from increased levels of viral accumulation in plant tissues. Thus MCMV suppressors may curb the RNA silencing mechanisms 
responsible for SCMV resistance (Xia et al. 2016). It is interesting that in MLN synergisms, SCMV is likely to possess important mechanism for enhanced infection by the co-infecting virus, MCMV, which is viewed as the primary disease behind MLN development. One of important proteins playing a significant role in the potyvirus infectivity is the HC-pro, which is multifunctional and possesses counter-defensive capacity to suppress the PTGS of the host. In the synergistic interaction with SCMV and MCMV, it is also likely that the accumulations of both MCMV and MCMV-derived siRNAs in maize that is reported to be increased remarkably compared to single infection implies that the presence of potyvirus was not only in favour of its own multiplication within the host but also catalyzing the multiplication of the partner co-infecting virus (Xia et al. 2016). In this case of synergism, the introduction of SCMV led to increased replication of MCMV hence the striking increase in symptom expression.

At the end of the experiment, mixed infections with MCMV and SCMV induced more severe symptoms than those observed in single viral infections regardless of the sequence of infection. Thus, there was no obvious difference in the expression level of the disease between SCMV+MCMV, MCMV+SCMV and MLN infected maize plants. Although there was a delay in the disease manifestation for the SCMV+MCMV infected plants but MLN disease was later observed in the co-infection.

In order to understand more on the interaction of maize plants with viruses causing MLN disease similar studies should be conducted-involving varieties that are resistance to either of the single viruses and see how they would respond to sequence of infection. In addition, it would be important to evaluate and understand the role of the individual viruses in the synergism.

\section{Acknowledgments}

This study was supported in part by grants from the Kenya Agricultural Productivity and Agribusiness Project (KAPAP) Collaborative Research Project and The Association for Strengthening Agricultural Research in Eastern and Central Africa (ASARECA) - MLND Project. Special thanks to the management of KALRO- Biotechnology Research Institute (BRI) Biotechnology Centre where the study was undertaken.

\section{References}

Anjos, J.R., Jarlfors, U. \& Ghabrial, S. A. (1992), "Soybean mosaic potyvirus enhances the titer of two comoviruses in dually infected soybean plants." Phytopathology 82, 1022-1027.

Anderson, E.J., Kline, A.S., Morelock, T.E. \& McNew, R.W. (1996), "Tolerance to black eye Cowpea mosaic potyvirus not correlated with decreased virus accumulation or protection from cowpea stunt disease", Phytopathology 80, 847-852.

Brantley, J.D. \& Hunt, A.G. (1993), "The N-terminal protein of the polyprotein encoded by the potyvirus Tobacco vein mottling virus is an RNA-binding protein." Journal of General Virology 74, 1157-1162.

Cabana, D., Watanabe, S., Higashi, C.H.V. \& Bressan, A. (2013), "Dissecting the mode of Maize chlorotic virus (Tombusvidae: Machlomovirus) transmission by Franliniella williamsi (Thysanoptera: Thripidae)", Journal of Economical Entomology 106, 16-24.

Calvert, L.A. \& Ghabrial, S. A. (1983), "Enhancement by Soybean mosaic virus of Bean pod mottle virus titer in doubly infected soybean", Phytopathology 73, 992-997.

Goldberg, K.B. \& Brakke M.K. (1987), "Concentration of Maize chlorotic mottle virus increased in mixed infections with Maize dwarf mosaic virus, strain B", Phytopathology 77, 162-167.

Goodman, R.M. \& Ross, A.F. (1974a), "Enhancement of potato virus X synthesis in doubly infected tobacco occurs in doubly infected cells", Virology 58, 16-24.

Goodman, R.M. \& Ross, A.F. (1974b). "Enhancement by potato virus $Y$ of potato virus $X$ synthesis in doubly infected tobacco depends on the timing of invasion by the viruses", Virology 58, 263-271.

Incarbone, M. \& Dunoyer, P. (2013), "RNA silencing and its suppression: novel insights from in planta analyses", Trends of Plant Sciences 18, 382-392.

Lommel, S.A., Kendall, T.L., Siu, N.F. \& Nutter, R.C. (1991), "Characterization of Maize chlorotic mottle virus", Phytopathology 81(8): 891-823.

Makone, S.M., Menge, D. \& Basweti, E. (2014), "Impact of Maize Lethal Necrosis Disease on maize yield: A Case of Kisii, Kenya", International Journal of Agriculture Extension 2(3), 211-218.

Manje, G., Biswanath, D., Makumbi, D., Babu, R., Semagn, K., Mahuku, G., Olsen, M.S., Bright, J.M., Beyene, Y. \& Prasanna, B.M. (2015), "Genome wide association and genomic prediction of resistance to maize lethal necrosis disease in tropical maize germplasm", Theoretical Application in Genetics 128, 1957-1968.

Nelson, S., Brewbaker, J. \& Hui, J. (2011), “Maize chlorotic mottle. University of Hawaii”, Plant Diseases 79, 6.

Rajama M. \& Valkonen J.P.T., 2009. "Control of nuclear and nucleolar localization of nuclear inclusion protein a of Picorna-like Potato virus A in Nicotiana species." The Plant Cell 21, 2485-2502.

Rochow, W.F. \& Ross, A.F. (1955), "Virus multiplication in plants double infected by potato viruses X and Y", Virology 1, 10-27. 
Savenkov, E.I. \& Valkonen, J.P.T. (2001), "Potyviral Helper-Component Proteinase Expressed in Transgenic Plants Enhances Titers of Potato Leaf Roll Virus but Does Not Alleviate Its Phloem Limitation”, Virology 283 (2), 285-293.

Syller, J. (2012), "Facilitative and antagonistic interactions between plant viruses in mixed infections", Molecular Plant Pathology 13, 204-216.

Uyemote, J.K., Claflin, L.E., Wilson, D.L. \& Raney, R.J. (1981), “Maize chlorotic mottle and Maize dwarf mosaic virus: effect of single and double inoculations on symptomatology and yield," Plant Disease 65, 39-41.

Vance, V.B. (1991), "Replication of potato virus X RNA is altered in coinfections with Potato virus Y", Virology 182, 486-494.

Vance, V.B., Berger, P.H., Carrington, J.C., Hunt, A.G. \& Shi, X.M. (1995), “59 proximal potyviral sequences mediate Potato virus X/potyviral synergistic disease in transgenic tobacco", Virology 206, 583-590.

Verchot, J. \& Carrington, J.C. (1995), "Evidence that the potyvirus P1 proteinase functions in trans as an accessory factor for genome amplification", Journal of Virology 69, 3668-3674.

Verchot, J., Koonin, E.V. \& Carrington J.C. (1991), "The 35-kDa protein from the N-terminus of the potyviral polyprotein functions as a third virus-encoded proteinase", Virology 185, 527-535.

Wangai, A., Redinbaugh, M.G., Kinyua, Z.M., Miano, D.W., Leley, P.K., Kasina, M., Mahuku, G., Scheets, K. \& Jeffers, D. (2012), "First Report of Maize chlorotic mottle virus and Maize Lethal Necrosis in Kenya", Plant Disease 96, 1582.

Xia, Z., Zhao, Z., Chen, L., Li M., Zhou, T., Deng, C., Zhou, Q. \& Fan, Z. (2016), "Synergistic infection of two viruses MCMV and SCMV increases the accumulations of both MCMV and MCMV-derived siRNAs in maize", Scientific Reports 6. Article number 20520. Doi:10.1038/srep20520.

Zhou, C.J., Zhang, X.Y., Liu, S.Y., Wang, Y.C., Li, D.W., Yu, J.L. \& Han, C.G. (2017), “Synergistic infection of BrYV and PEMV 2 increases the accumulations of both BrYV and BrYV-derived siRNAs in Nicotiana benthamiana", Science Report 7, 45132. 